\title{
Evaluation of Shared Space to Reduce Traffic Congestion
}

\author{
Colin Frosch, ${ }^{1}$ David Martinelli, ${ }^{2}$ and Avinash Unnikrishnan ${ }^{3}{ }^{3}$ \\ ${ }^{1}$ Kimley-Horn, Raleigh, NC, USA \\ ${ }^{2}$ Department of Civil and Environmental Engineering, West Virginia University, Morgantown, WV 26506, USA \\ ${ }^{3}$ Department of Civil and Environmental Engineering, Portland State University, Portland, OR 97207, USA
}

Correspondence should be addressed to Avinash Unnikrishnan; avinashu@gmail.com

Received 27 December 2018; Accepted 26 May 2019; Published 13 June 2019

Academic Editor: Ludovic Leclercq

Copyright (c) 2019 Colin Frosch et al. This is an open access article distributed under the Creative Commons Attribution License, which permits unrestricted use, distribution, and reproduction in any medium, provided the original work is properly cited.

\begin{abstract}
Pedestrian and vehicle interactions often lead to conflicts that bring about safety, traffic congestion, and priority or right of way issues. Common methods used in the past to combat said issues have largely relied on the principle of separating the motions of pedestrians and vehicles by means of bridges, tunnels, signals, and access restrictions. A different approach known as shared space aims to solve the same problems with a less structured and defined environment which instead places more reliance on human interaction and perception. Although it has been used in multiple scenarios across Europe with success, instances of shared spaces in the United States are few. In the past, the success of shared space has mainly focused on safety, aesthetic, and pedestrian use metrics, with little quantitative knowledge regarding the traffic congestion relief benefits. This research focuses on evaluating and quantifying the traffic congestion relief abilities of shared space designs utilizing Vissim traffic microsimulation software and the economic impact these changes can make. A major pedestrian crossing location on West Virginia University's Downtown campus along a major urban arterial was chosen as the case location upon which the model was to be built. This location posed unique aspects, which made it a prime choice for this research as the major concern for years has been traffic congestion, in addition to pedestrian safety and aesthetic appeal. The results of the analysis show that shared space can reduce vehicle travel time by up to $50 \%$ and delays by $66 \%$.
\end{abstract}

\section{Introduction}

Shared space traffic designs have been used in an increasing number of countries around the world to solve congestion, safety, accessibility, and community issues. This design concept was first pioneered in the Netherlands by Hans Monderman in the late 1900s, but has since been adapted to fit other case-specific areas within Western Europe and most recently in North America. The applications of shared space range between urban and suburban locations and have been found most suitable for areas used by multiple modes of transport $[1,2]$.

Although the specific implementation details of each shared space have varied greatly across time and location, the core features of ridding an area of most if not all traffic signs, demarcations, and traditional controls, to allow multimodal users more independence and less decision making reliance on these engineered elements have been presented. By forcing drivers and pedestrians to no longer rely on traffic signals, signs, and designated crosswalks to create distinct rules of priority, they must instead be more alert to their surroundings and communicate with other users. Users quickly realize this upon entering a shared space environment and begin to proceed with more caution and make more eye contact between users. These actions by the individual user have been scientifically shown to increase the pedestrian safety and decrease the average vehicle speeds $[1,2]$. It has also been noted anecdotally that the congestion and travel time through a specified area has been reduced as result of a shared space implementation; however, the data to back up these claims is rare. Finally, shared space designs have been shown to greatly increase the public's perception of an intersection, corridor, or locale through the use of improved aesthetic elements. These now more welcoming areas have also in turn experienced improved and revitalized economic markets brought about by an increase in mostly bicycle and pedestrian users to the area $[3,4]$. 
As mentioned above, there is an untapped potential with shared space to address congestion and traffic delay issues that arise at multimodal intersections or corridors. The traffic flow dynamics based on user behavior within a shared space have the potential to reduce this congestion and delay problem. Municipalities and design firms have unfortunately not yet turned to shared space as a viable means of addressing congestion problems. A simple, yet effective, way to examine traffic efficiency effects of an implemented shared space design is needed if shared space is to be taken seriously by engineers, planners, government officials, and community members.

The objective of this research is to evaluate the traffic congestion and vehicular delay impacts provided by a shared space design alternative compared to a traditional design. The research develops a microsimulation traffic modelling method using existing tools which would provide sufficient information to be used in decision making processes based on metrics such as travel time and delay. We present a novel way to adapt PTV Vissim's existing capabilities to obtain a conservative estimate of the impact of shared space on congestion levels. A case study location known as Grumbein's Island, a major pedestrian crossing on the West Virginia University downtown campus, was chosen for its widely known vehicular congestion and delay problem stemming from the very cyclical pattern of student pedestrians crossing at a single location. Vehicular and pedestrian traffic volume as well as turning movement percentages was collected in order to create a realistic model in PTV Vissim. In addition to the data collected, satellite images of the current roadway design and a conceptual design drawing are used as a baseline for the PTV Vissim model. This model is then used to extract travel time and delay in order to assess the impact of a shared space design.

\section{Literature Review}

Since various types of shared space projects have been emerging in several countries, engineering and research studies are now emerging with some regularity. The table, Table 1 , provides an overview of the benefits observed at shared space implementations in Europe and the United States.

In addition, quasishared space like zones can be observed in major cities such as Barrack Street in Sydney, Chapel Road in Bankstown, and Jack Mundey Place at The Rocks in Sydney [5]. Jordan is looking to implement shared space in roads such as Al Medina Street in Amman which historically have had a healthy pedestrian automobile mix but have lost their identity to improve vehicular traffic [13].

Note that in almost all the above implementations, shared space has resulted in improved pedestrian safety which might be attributed to speed reductions [14, 15]. Monderman's theory that at lower speeds, pedestrians and drivers would be able to establish eye contact and "socially interact" to anticipate each other's behavior and determine their own appropriate response has been successful in reducing accidents and injuries [16]. Past implementations also show that shared space has also been successful in both busy streets in urban areas as well as rural areas. Shared space is also associated with reductions in most types of traffic conflicts [7]. In certain cases, e.g., Poynton, shared spaces have led to improved traffic flow. However, to date, there exists little to no published work documenting the quantification of the congestion benefit (or lack thereof) of shared space, which is the main focus of this paper.

\section{Microsimulation Model}

Some researchers have developed microsimulation models, based on social force theory, to model shared space [1719]. Although the methodology used to create said models could be replicated and perhaps expanded to measure traffic parameters, it has been deemed to be too complex to be generalized. Our goal is to develop a framework which can be used by transportation designers and planners to measure the congestion impacts of shared space. A modeling technique which can capture the shared space dynamics, while also being available to simulate more traditional designs within a small network, is needed in the industry to fill the chasm between current methods and what is required to advance shared space as a viable alternative. With this in mind, PTV Vissim was chosen to be the platform for this model due to its comprehensiveness, industry prevalence, reputation, and flexibility. However, Vissim (and all other microsimulation platforms) is not explicitly capable of modeling shared space; therefore, adaptations to it are necessary.

Within PTV Vissim, the current standard to determine right of way between vehicles, pedestrians, or vehicles and pedestrians is by using conflict areas or priority rules. Both methods allow the modeler to dictate which direction of flow has priority over the other. The flow without priority will then yield to the other movement of traffic. There is also the option of not placing any rules regarding priority in the simulation which results in simulated users not seeing each other and behaving as such. The last option exists only within the conflict area tool and allows one to express potential conflicts to the simulated users, but that it is not defined. In this case, simulated users can see the other users and know that their movement will conflict. They are then left to their own devices to decide which user shall proceed first. In the model, this is determined by multiple metrics that are either measured or randomly assigned to drivers and pedestrians, such as which user arrived first, vehicle speed, distance away from the potential conflict, level of driver/pedestrian aggression, etc. These metrics are the same as those used in the social force approach models discussed earlier [17-19] and the variables that real-life shared space users would encounter in order to make a decision as either a pedestrian or driver. These factors make this approach the closest and most suitable for simulating shared space. When this undetermined priority was placed between a single vehicle and single pedestrian, the users behaved nearly identically as if the pedestrian was given priority. In the literature review, it was noted that previous models and field data both show that shared space interactions between vehicles and pedestrians can be summarized by vehicles staying on course and only accelerating or decelerating to 
TABLE 1: Observed benefits of shared space implementation.

\begin{tabular}{|c|c|}
\hline Location & Benefits \\
\hline $\begin{array}{l}\text { Oudehaske and Makkinga, } \\
\text { Netherlands }[1,2]\end{array}$ & $40 \%$ reduction in vehicle speed \\
\hline Drachten, Netherlands [5] & Reduction in accidents from an average of 11 per year to only 2 in the first year \\
\hline Bohmte, Germany [6] & $\begin{array}{l}\text { Reduction in accidents on a busy street }(12000 \mathrm{vpd}) \text { from one per week to none in } \\
\text { four weeks }\end{array}$ \\
\hline Poynton, England $[3,4]$ & $\begin{array}{c}\text { Average speed fell to } 20 \mathrm{mph} \text {, travel time decreased and congestion improved, safety } \\
\text { improved with one minor accident in the first } 3 \text { years compared to } 4-7 \text { in previous } \\
\text { years, and economic revitalization with } 80 \% \text { of retailers reporting increased } \\
\text { turnover }\end{array}$ \\
\hline $\begin{array}{l}\text { Exhibition Road, London, } \\
\text { England [7] }\end{array}$ & Reduction of number and severity of conflicts \\
\hline Graz, Austria [8] & $\begin{array}{l}\text { Reduction in vehicular speeds, improvement of social interactions and awareness, } \\
\text { and no reported accidents in first four months }\end{array}$ \\
\hline Noordlaren [9] & Reduction of speeds by 6-7 mph \\
\hline $\begin{array}{l}\text { Bell Street Park, Seattle; } \\
\text { Davis Street, Portland; } \\
\text { Santana Row, Promenade, } \\
\text { San Jose, USA [10] }\end{array}$ & Low vehicle speeds, improved safety \\
\hline Cambridge, MA [11] & Increased pedestrian activity and improved vibrancy \\
\hline $\begin{array}{l}\text { Market Square, Pittsburgh } \\
{[12]}\end{array}$ & Improved business and social activity \\
\hline
\end{tabular}

avoid collisions. Note that pedestrians perform the opposite behavior and vary their route to avoid collisions but remain at a constant speed. Since pedestrians are unable in PTV Vissim to stray from their link's path, and the vehicles would always yield to them, it was deemed that this undetermined conflict area was the appropriate and conservative option when attempting to estimate the traffic delay, but could also be replicated with the pedestrians having outright priority and resulting in similar outcomes.

Finally, the lane/link width for vehicles was reduced to $6 \mathrm{ft}$. in order to reduce the distance between pedestrians and vehicles to trigger a conflict. This allows vehicles in the model to only yield to pedestrians when a collision will occur, rather than yielding to all pedestrians in the lane or crosswalk, even when a collision will not occur. The result is a smaller headway between vehicles and pedestrians that exists in real-world shared space scenarios. Likewise, the pedestrian links were also reduced in width to represent the width of 1-2 pedestrians crossing rather than an entire width of a crosswalk, therefore better representing the space they actually occupy.

Theoretically in a shared space environment, pedestrians are free to cross the street in whichever path they desire. This lack of designated crossing points leads to an infinite number of O-D pairs and crossing points that would need to be addressed in the model. Knowing, however, that pedestrians will ordinarily take the shortest possible path, we can reduce the number of crossing points necessary to include in the model by identifying major origins and destinations and mapping the crossing paths between them. Research has shown that in a real shared space scenario, vehicle, bicyclist, and pedestrian routes tend to cluster around a limited number of crossing paths [18]. This knowledge allows the modeler to choose how many pedestrian crossing paths, and where to place them, in the PTV Vissim model, based on knowledge of current pedestrian trip generators. The number of paths required to simulate the shared space as closely as possible will vary between sites based on the surrounding environment. Note that the pedestrian routes chosen should represent the shortest path for a pedestrian group and will therefore be more likely to cross the space at an acute angle rather than at a perfect ninety-degree angle (as is typical for designated crosswalks). The combined paths modeled in PTV Vissim should characterize the desired movements of the majority of the pedestrian users.

It should also be noted that the spacing of the pedestrian crossing paths is of critical importance. Due to the setup of the PTV Vissim software, there must be sufficient space on a vehicular travel link between two neighboring pedestrian crossing points to accommodate the largest class of vehicles being modeled. Innately, PTV Vissim does not allow a vehicle to cross a pedestrian path (conflict point) until it knows that the vehicle can traverse that conflict point without being stopped at the next one and consequently block the preceding conflict point. Therefore, without enough space for a vehicle to stop between two conflict points, the vehicle must wait until both conflict points are clear. This does not mimic the real workings of a shared space, and therefore it is imperative that there be enough space on each vehicular link between neighboring conflict points for the largest vehicle to stop. This layout of pedestrian crossing paths allows vehicles to incrementally progress through the intersection as they would in a real shared space. 


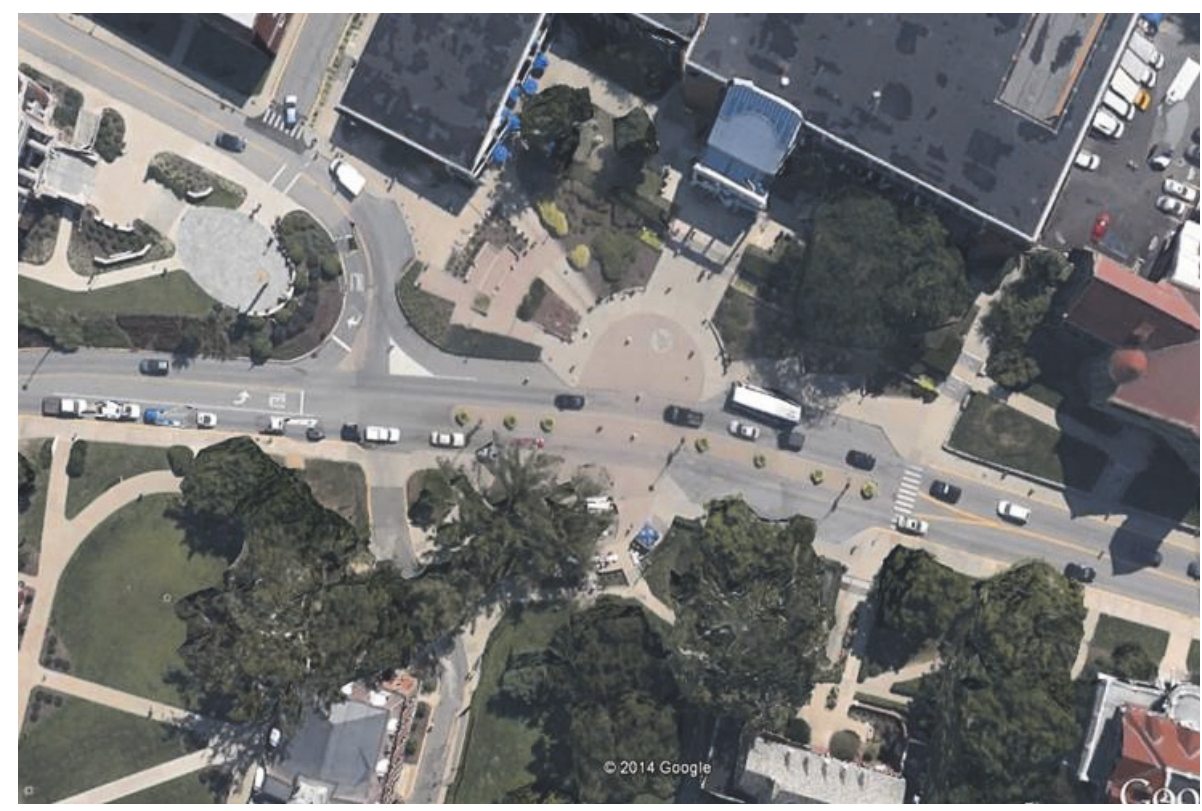

FIgURE 1: Current Grumbein's Island configuration on University Avenue in Morgantown, WV (Google).

Another main change which was applied in comparison to a traditional traffic model in Vissim was the vehicular speeds. In this case, our research points us to the fact that shared space designs, even without the use of posted speed limits, will reduce observed vehicle speeds to the range of 10$20 \mathrm{mph}$. Depending on the location and dynamic aspects of vehicle and pedestrian volume, the speed at any given shared space can vary between these two ranges. Since this model will be used to test the congestion relief aspects of shared space, the more conservative value of $10 \mathrm{mph}$ was chosen.

\section{Case Study}

The case study location selected is located on the Downtown campus of West Virginia University in Morgantown, WV, as shown in Figure 1. WVU is currently home to approximately 30,000 students and another 30,000 citizens within the city limits. There are two main arteries, Beechurst and University Avenues, which run in the general North-South direction within the Downtown campus. University Avenue bisects the Downtown campus of WVU with many of the freshman dorm facilities as well as the student union, known as the Mountainlair, on one side, and the majority of the academic buildings on the opposite side of the street. Therefore, a pedestrian crosswalk was necessitated, and in the 1930s, a pedestrian island and single unsignalized crosswalk were installed at this location under the direction of then facilities manager and professor Dr. Grumbein to facilitate the safe crossing of students, faculty, and citizens. As the student population has greatly increased over the past 80 years, this location now known as Grumbein's Island, experiences daily congestion and traffic delays for drivers on University Avenue.

The arrangement of the WVU facilities with one main "crosswalk" on University Avenue results in a large number of pedestrians crossing University Avenue directly in front of the Mountainlair for a 10-20-minute period between classes every hour on Monday, Wednesday, and Fridays and approximately every 90 minutes on Tuesdays and Thursdays. The influx of pedestrians to a single unsignalized crosswalk causes drivers to stop and wait for an extended period of time as the headway between pedestrians is typically insufficient to drive through. Long vehicle queues begin to form rapidly at this crosswalk as the rate of vehicles entering the queue from other streets is much greater than the rate at which cars can cross this single crosswalk. After the approximately 20minute period is over, the queue begins to recede until normal traffic flow is resumed after an additional 10-20 minutes pass. This means that at multiple times during the day, there is an almost 40-minute period every hour in which traffic on this street is backed up, moving slowly, or potentially stopped for an extended period of time.

During the weeks of March 23rd-30th and March 30thApril 6th, 2014, a two-week-long data collection period was undertaken to provide base data to create a model of the current scenario and base the parameters for the shared space model on. Mounted radar vehicle counters and manual turning counters, operated by a group of volunteers, were used to collect the data during this collection period.

Figures 2 and 3 present a summary of the data collected during this first data collection period, which included vehicle volumes during peak and nonpeak hours, vehicle turning ratios at intersections, as well as pedestrian volumes on a 15-minute time interval. This out-of-the-ordinary pedestrian volume time period was chosen to capture the unique volume changes over the course of time at a university campus. More details of the data are provided in [20].

Once the PTV Vissim model was created for the current configuration, and travel time values were measured in 


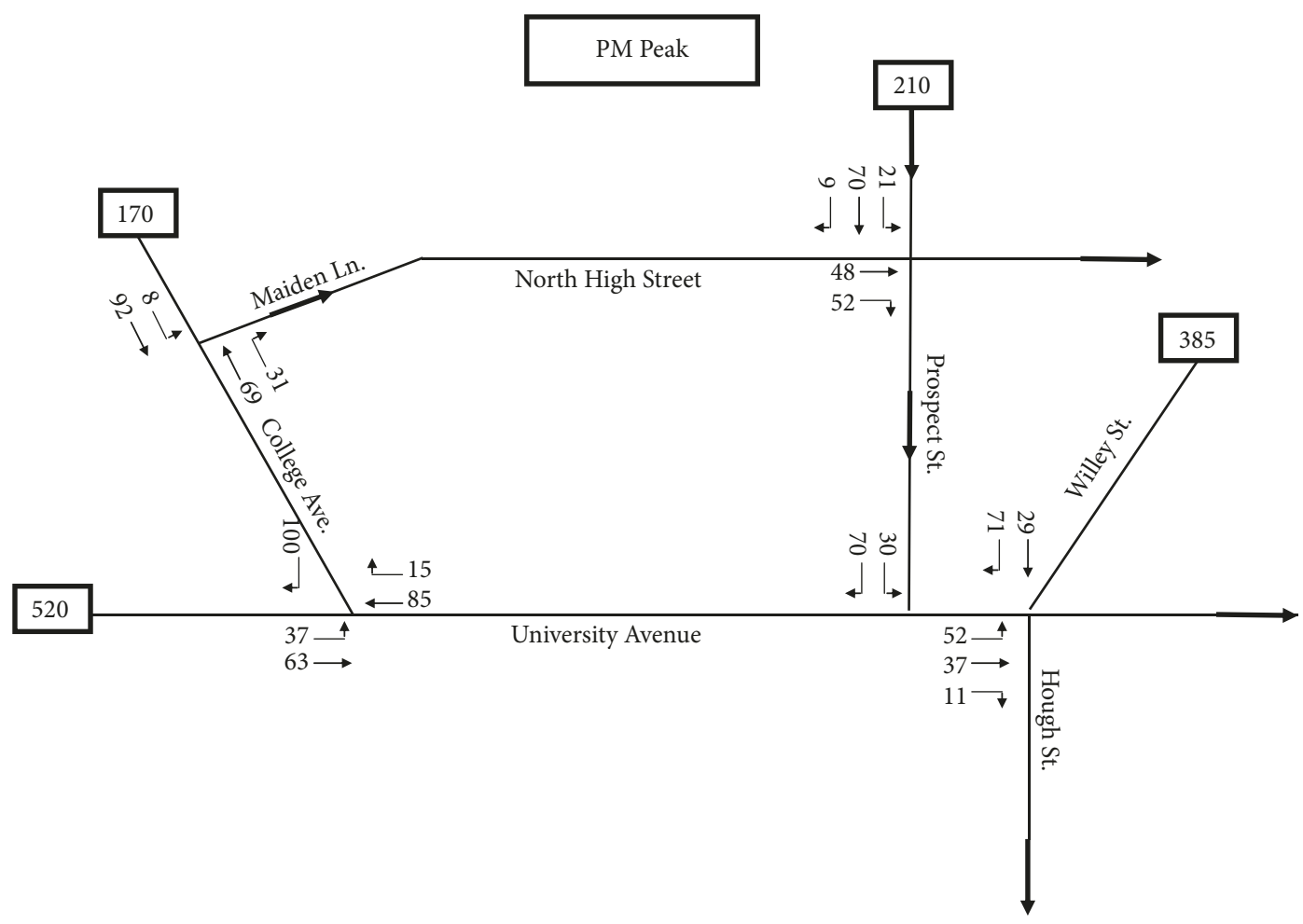

Figure 2: Traffic volume.

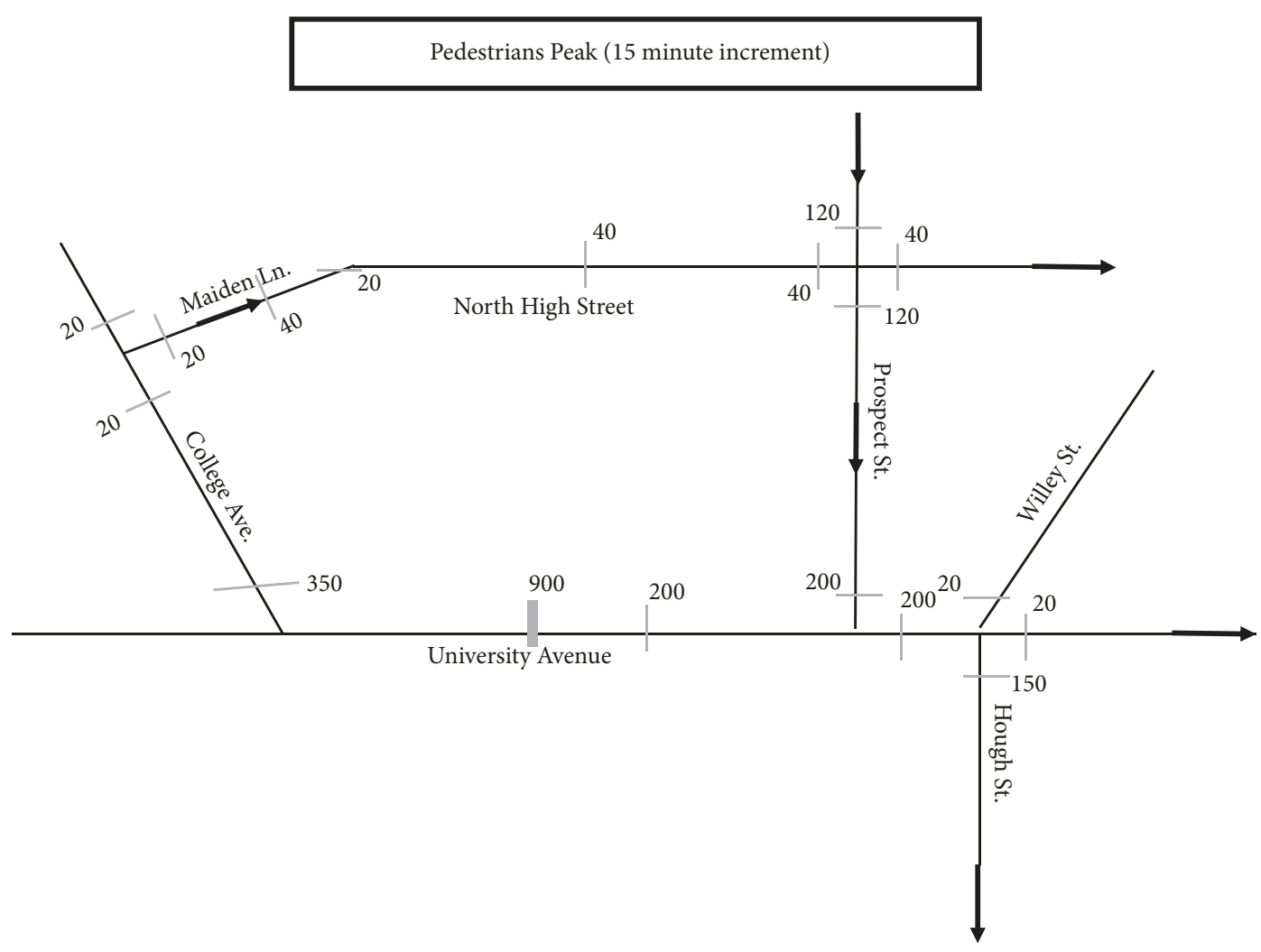

Figure 3: Pedestrian volume. 


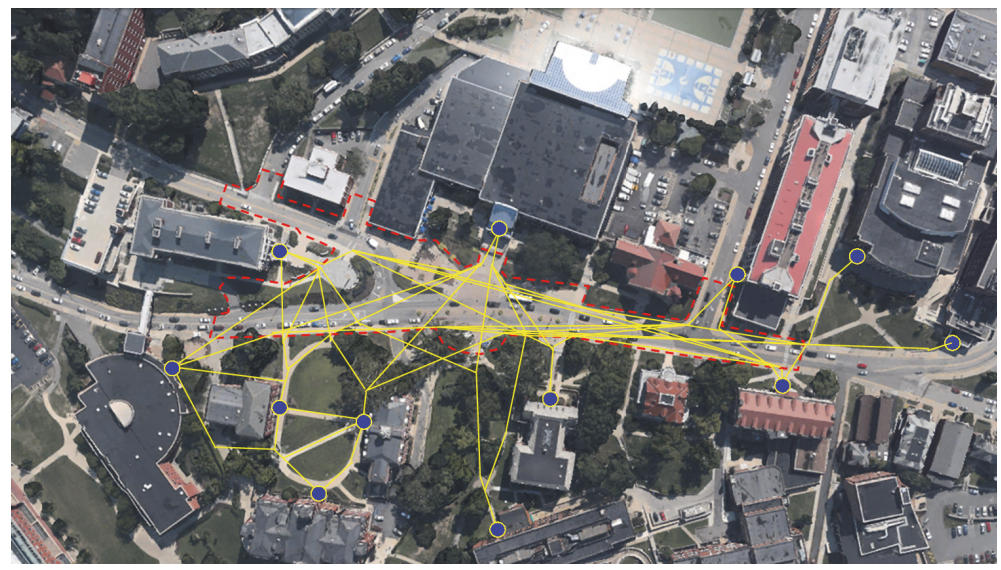

FIGURE 4: Forecasted pedestrian O-D pairs (blue: origin/destination, yellow: routes, and red: shared space boundary) (Google).

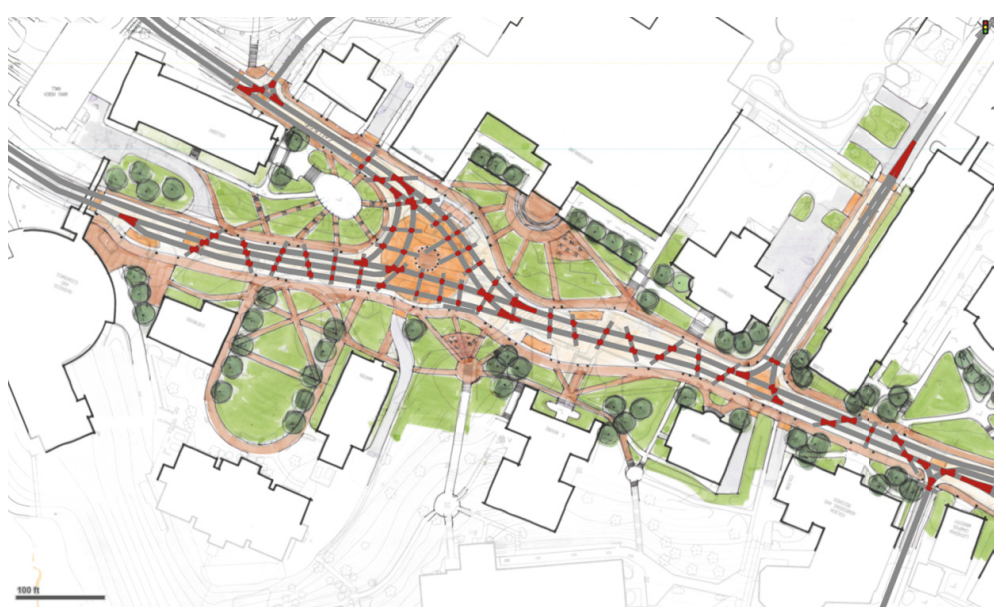

FIgURE 5: Designated conflict areas in PTV Vissim simulation.

the model, in-person trial travel time runs were taken to verify the model. This verification process was a success and warranted further progress on the model to now include the shared space design.

To identify pedestrian routes, major origin and destination locations were identified in the near vicinity of the shared space boundaries. In the case of WVU's campus, this correlated to mainly academic and student service facilities. Pedestrian routes were then transcribed on to a satellite image connecting these designated origins and destinations. Pedestrian routes first followed sidewalks and pathways to get to the approximate boundary of the shared space and then were made to have a single straight line crossing of the street to the chosen destination. The resulting web of $\mathrm{O}-\mathrm{D}$ pedestrian pairs is shown in Figure 4.

In collaboration with WVU administrators and Stantec Consulting Services Inc., a conceptual design rendering was created and used as the foundation for the geometric layout of the model in PTV VISSIM as shown in Figure 5.

We created multiple different scenarios of pedestrian dispersion for a total of six shared space simulation iterations. First, three different pedestrian volume dispersions over the four 15-minute time periods were created placing varying levels of stress on the shared space design during the peak 15-minute period. The first configuration was based on data and conclusions made by observing pedestrians on two separate occasions at the site. The second two configurations shifted the peak 15-minute time period intensity slightly. The second level of variation was provided by altering the ratio of preferred routes between origins and destinations that pedestrians would take. This varied the individual volume on each pedestrian link. By combining the two sets of scenarios, with three time variations and two route variations, respectively, we were able to make a total of six scenarios to test the model.

\section{Analysis of Results}

The analyses were based on travel time and delay which can be easily obtained from the PTV VISSIM simulation platform. The results shown below are for the 60-minute PM peak. The trends were consistent for the AM peak also.

Figure 6 shows the average travel time across both directions. We can see that the average travel time was lower 


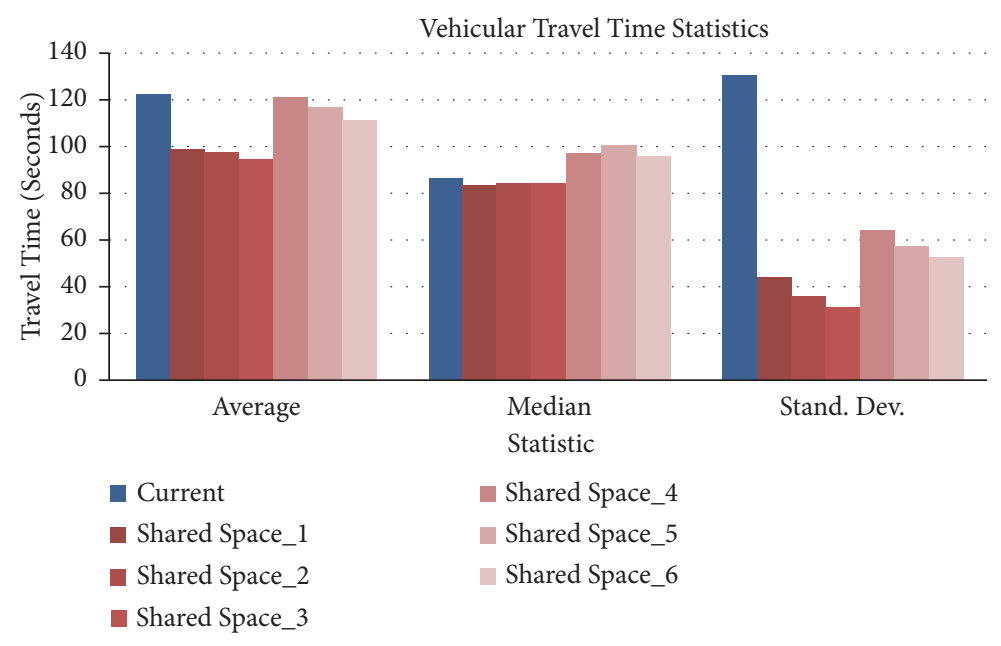

Figure 6: Vehicle travel time statistics.

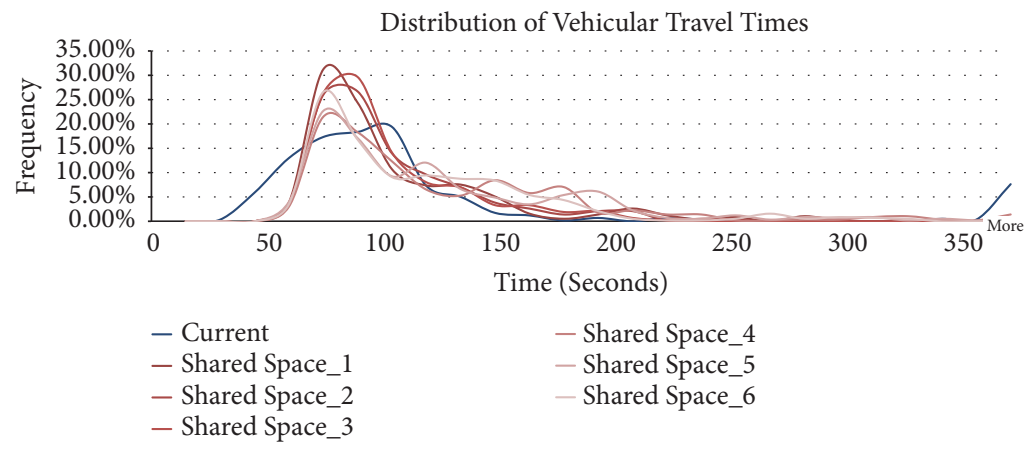

FIGURE 7: Distribution of vehicular travel time.

for all 6 iterations of the shared space simulation compared to the current traffic scenario. On average, across the six shared space simulations, the travel time for vehicles decreased by $13 \%$ and by $19 \%$ for Simulation 1 which represents the best estimate of pedestrian behavior. We can see that the standard deviation drastically decreased from the current scenario to all six of the shared space simulations. This equates to the risk of a driver not being able to traverse the prescribed area within the average time. The variance of the shared space travel time is significantly lower than that of the current scenario, meaning drivers could expect a much more consistent commute within the shared space. The current scenario had multiple outliers which stretched the variance of the observed travel time data to an extreme extent.

Figure 7 exhibits the presence of extreme outliers, where $7.63 \%$ of all vehicles traveling along University Avenue in the current model incurred a total travel time of more than 375 seconds. Compare this to the shared space simulations which have barely any occurrences above 200 seconds of travel time across all six models. It can also be seen that minimum travel time, or free flow travel time, for the current scenario is lower than the shared space simulations. Remember that this is due to the inherently lower speed limit set as described in the methodology within the shared space models at half of the current speed limit. This short free flow travel time however is counterbalanced by the extreme outliers on the other end of the spectrum. Therefore, in the current scenario, drivers have the chance of experiencing very little traffic and getting through quickly, but risk of being stuck in the queue for a long time if they do hit the inevitable traffic. On the other hand, in the shared space simulation, it is almost certain that a driver's travel time would be within a much smaller range.

Figure 8 summarizes the vehicular travel time statistics for the peak pedestrian period. First looking at the average travel time, we can see that the first shared space model decreased by more than 170 seconds. On average, the shared space models decreased the travel time by 166 seconds, which represented $54 \%$ of the current travel time for vehicles during the peak pedestrian period. The median and standard deviation averaged across all six shared space models also showed significant drops of $42 \%$ and $72 \%$, respectively. Figure 9 shows the distribution of travel time occurrences during the peak pedestrian period. We can see here that more than $40 \%$ of drivers observed in this time period experienced a travel time exceeding 375 seconds in the current model. On the other hand, some of the shared space models had no observed travel times above 250 seconds. The models that did had very few drivers above this range.

In addition to analyzing and comparing the raw travel time data from the seven separate models, a PERT analysis 


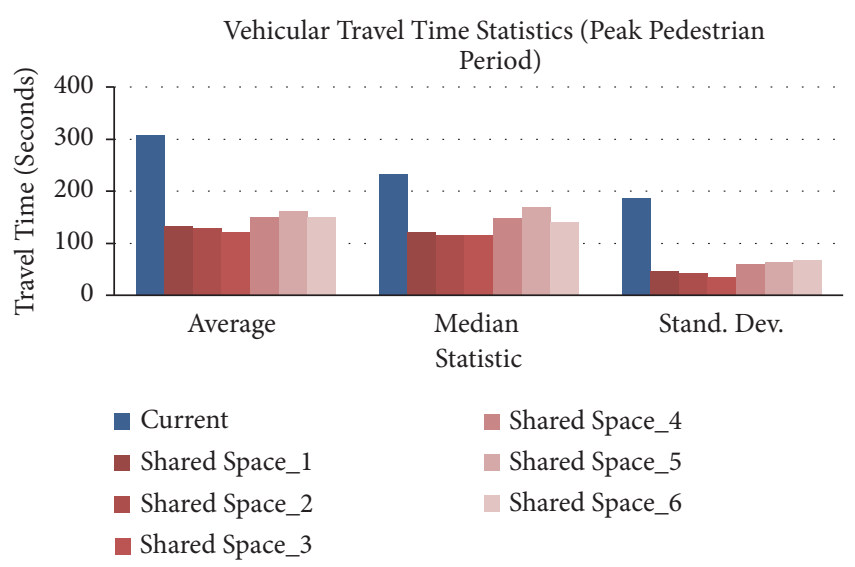

FIgURE 8: Vehicular travel time statistics (peak pedestrian period).

was also performed. The PERT technique, or Program Evaluation and Review Technique, is typically used in project management applications to gain a better understanding for the expected duration of a project or program. This projected duration is calculated using the minimum, mode, and maximum duration expectations in a weighted average format as shown in the equation below:

$$
\begin{aligned}
& \text { Mean or Expected Duration } \\
& \quad=\frac{\text { minimum }+4 * \text { mode }+ \text { maximum }}{6}
\end{aligned}
$$

The calculation results indicated that the current scenario would have a mean or expected travel time of 200 seconds. Comparatively, the shared space model PERT expected travel times ranged between 107.5 and 127.5 seconds, with an overall average expected value of 115.4 seconds. That is an 84.6second drop, or $42.3 \%$ decrease, in expected travel time from the current operation.

Figure 10 summarizes the statistics for the vehicular delay average over the entire 60 simulation periods. We can see that the average delay decreased by nearly $50 \%$ for the average shared space model and by $56 \%$ for the primary shared space model. The resulting average delay for all six shared space models is 43.57 seconds across the entire study area. It should also be noted that the median and standard deviation also decreased significantly, and more so than the travel time. By evaluating the distribution of observations in Figure 11 for the vehicular delay, we can see how all the observations from the six shared space models are highly congregated towards the lowest bin at 15 seconds. The remaining shared space observations tail off quickly, with only a rare occurrence of an observation above 150 seconds. In the current operation, on the other hand, the highest percentage of observations does not fall until the 60- and 75-second bins with approximately $19 \%$ of the observations in each. The observations for the current operation also tail off quickly up to the 150 -second point; however, there is again the presence of a large group of extreme outliers. Just like for the travel time statistics, 7.63\% of the observed vehicles experienced a travel delay in excess of 375 seconds.
The PERT analysis was again performed on the delay data observed in the models. The current model expected delay was found to be 157.5 seconds. The six shared space models returned PERT expected delay values ranging between 37.5 and 67.5 seconds, with an average of 54.6 seconds. This represents a 102.9-second, or 65.34\%, drop in delay between the current and shared space designs. Again, this PERT calculation helps show the significant advantage of utilizing shared space in this scenario.

As we know through experience, Morgantown is a very dense network of urban streets meeting at signalized and stop-controlled intersections. Our hypothesis through observation is that Grumbein's Island location was not only a catalyst for congestion and delay for drivers along University Avenue but also throughout the downtown network. This is evidenced by the long queues exhibited in the current model. These queues would often extend beyond the study area and into surrounding intersections setting off a chain effect leading closer to gridlock. Shared space on the other hand drastically reduced the queue lengths, therefore taking away the direct interaction between intersections and reducing the spread of congestion and grid lock. The slower speeds of vehicles in a shared space also help reduce the speed and promulgation of traffic waves through the system. To truly capture these effects, a larger more complex model is needed, but our current results certainly are in support of these predictions.

\section{Conclusions}

This study has successfully shown how simulation software such as PTV Vissim can be utilized to simulate and analyze shared space as a proposed solution for traffic congestion problems. By taking advantage of built-in functionalities within the existing PTV software package, the planner or engineer can replicate these efforts to assess unique shared space designs. While several studies have documented the efficiency and safety improvements from a pedestrian perspective, to the best of our knowledge, this study is among the first to quantify the potential congestion benefits of shared space. A major pedestrian crossing location on West Virginia University's Downtown campus along a major urban arterial was chosen as the case location. This location posed unique aspects which made it a prime choice for this research as the major concern for years has been traffic congestion, in addition to pedestrian safety and aesthetic appeal. The results of the analysis show that shared space can reduce vehicle travel time by up to $43 \%$ and delays by $66 \%$. Shared space was also found to improve the reliability of travel times and hence reduce the chances of being stuck in traffic for longer periods.

Limitations of modeling shared space using PTV Vissim were also noted in this study. In order to define the routes of vehicles and pedestrians, limited discrete origindestination pairs needed to be defined. In scenarios like the case study, this was feasible based on the limited amount of trip generators on the periphery of the shared space for pedestrians and the intersection of only two main streets for vehicles. Theoretically, if a shared space had multiple 


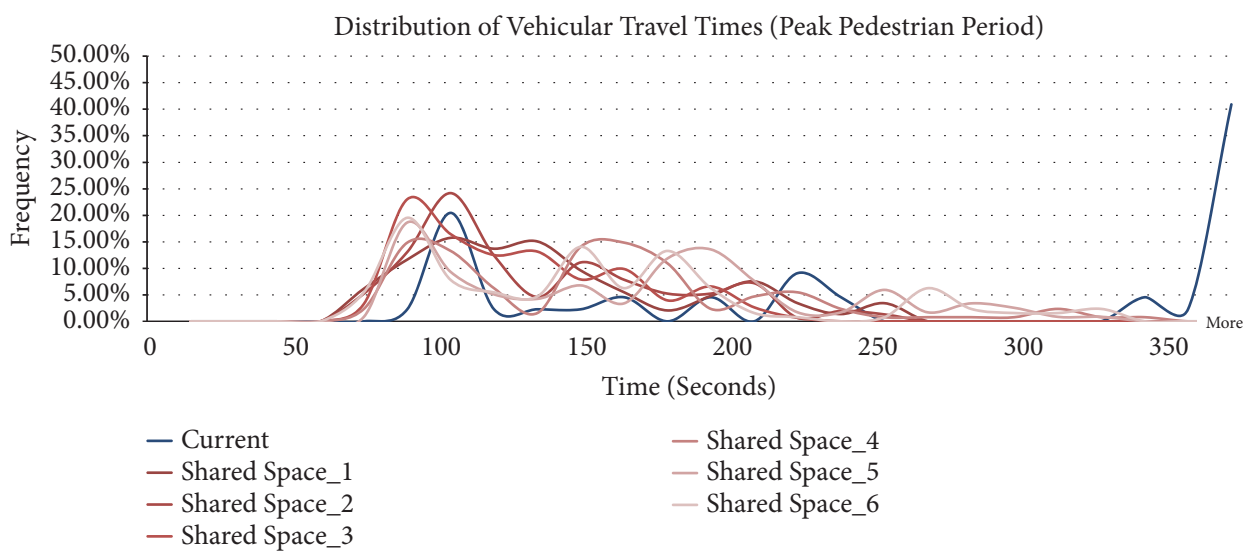

FIGURE 9: Distribution of vehicular travel time during peak pedestrian period.

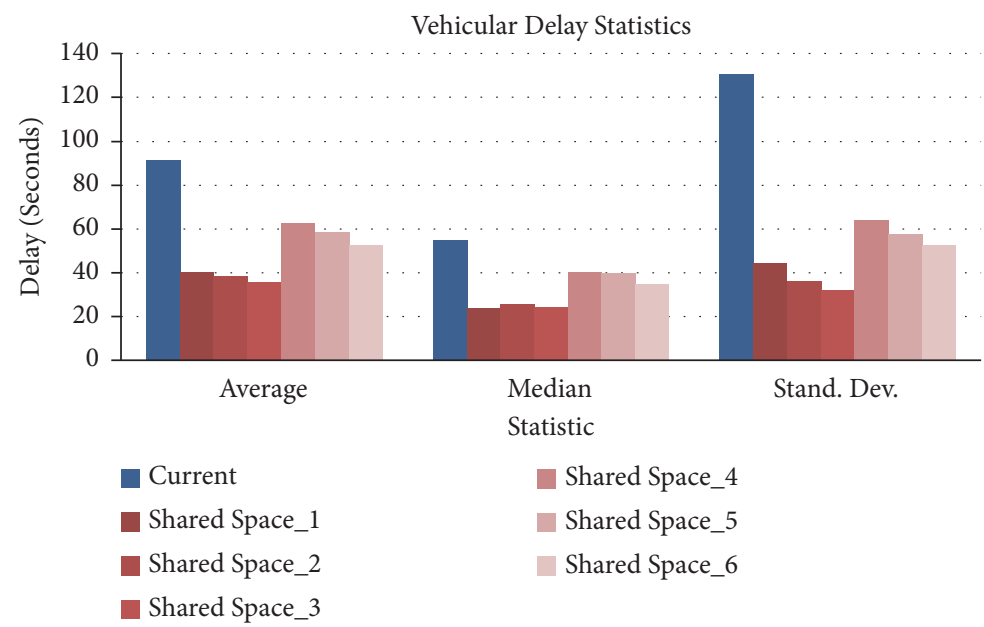

FIgure 10: Vehicular delay statistics.

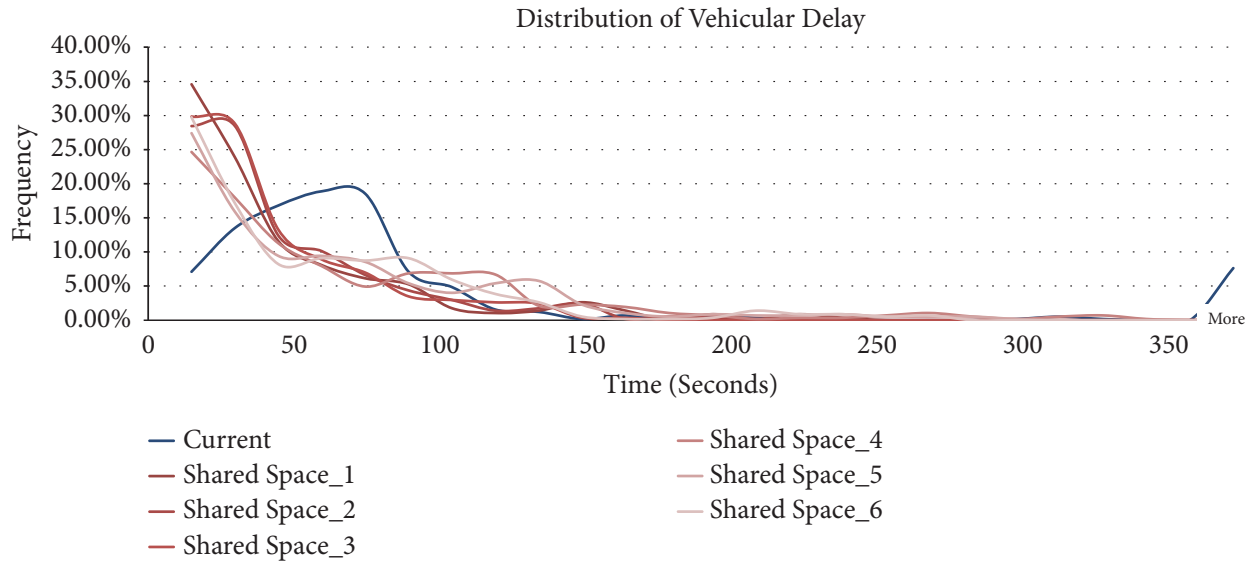

FIGURE 11: Distribution of vehicular delay.

pedestrian or vehicular routes that were too numerous to space appropriately within the confines of the model as discussed previously, they would need to be combined which would skew the results. This scenario is not likely to occur in the real world though. As found in the literature, most shared spaces occur along vehicular corridors where there is a set route for drivers or at intersections with a few intersecting roads. Likewise for pedestrians, the origins and destinations are ordinarily set by store fronts at a minimum which would be spaced sufficiently far apart to allow modeling in PTV 
Vissim. Bicycle users were also not incorporated into this model as they were not observed to constitute a significant portion of the total users. If bicycles were incorporated, it is anticipated that the overall traffic flow and resulting travel time and delay statistics would vary as bicycle users in a shared space share some of the characteristics of both pedestrians and vehicles.

\section{Data Availability}

The simulation and analysis data used to support the findings of this study are available from the corresponding author upon request.

\section{Disclosure}

Any error, mistake, or omission related to this research paper is the sole responsibility of the authors.

\section{Conflicts of Interest}

The authors declare that there are no conflicts of interest regarding the publication of this paper.

\section{Acknowledgments}

The authors would also like to acknowledge the Dwight David Eisenhower Transportation Fellowship Program (DDETFP) for funding this work through DDETFP Graduate Fellowship.

\section{References}

[1] B. Hamilton-Baillie, "Shared space: reconciling people, places and traffic," Built Environment (1978), vol. 34, no. 2, pp. 161-181, 2008.

[2] B. Hamilton-Baillie, "Towards shared space," URBAN DESIGN International, vol. 13, no. 2, pp. 130-138, 2008.

[3] M. Express, "Traders share in success of poynton shared space road scheme," http://www.macclesfield-express.co.uk/news/ local-news/traders-share-success-poynton-shared-2526261, 2013.

[4] A. Kirkup, "Poynton town centre," Institution of Civil Engineers, 2013, https://www.ice.org.uk/disciplines-and-resources/casestudies/poynton-town-centre.

[5] A. Gillies, "Is the road there to share? Shared space in an Australian context," University of New South Wales, Bachelor of Planning, 2009.

[6] C. Whitlock, "A green light for common sense," The Washington Post Foreign Service, 2007, http://www.washingtonpost.com/ wp-dyn/content/article/2007/12/23/AR2007122302487.html.

[7] I. Kaparias, M. G. H. Bell, W. Dong et al., "Analysis of pedestrian-vehicle traffic conflicts in street designs with elements of shared space," Transportation Research Record: Journal of the Transportation Research Board, vol. 2393, pp. 21-30, 2013.

[8] W. Fischer, "Shared space in graz (austria)," Eltis, http://www .eltis.org/discover/news/shared-space-graz-austria-0, 2011.

[9] Allianz, "Why making streets risky improves road safety," https://www.allianz.com/en/about_us/open-knowledge/topics/ mobility/articles/-why-making-streets-risky-improves-roadsafety.html/, 2012.

[10] G. Behrens, "Sharing the street: shared space in an american context," University of Washington, Urban Planning, 2014.

[11] P. Langdon, "US shared space: starting small," Better Cities \& Towns, 2010, http://bettercities.net/article/us-shared-spacestarting-small-13673.

[12] T. Snyder, "Bikes, cars, and people co-exist on pittsburgh's shared streets," Streetsblog USA, 2014, http://usa.streetsblog.org/ 2014/07/03/bikes-cars-and-people-co-exist-on-pittsburghsshared-streets/.

[13] M. Tawil, C. Reicher, K. Z. Ramadan, and M. Jafari, “Towards more pedestrian friendly streets in jordan: the case of al medina street in amman," Journal of Sustainable Development, vol. 7, no. 2, pp. 144-158, 2014.

[14] W. A. Leaf and D. F. Preusser, Literature Review on Vehicle Travel Speeds and Pedestrian Injuries Among Selected Racial/Ethnic Groups, U. S. Department of Transportation: National Highway Traffic Safety Administration, Washington, DC, USA, 1999.

[15] C. Gilman and R. Gilman, Shared-Use Streets - An Application of "Shared Space" to an American Small Town, 3rd Urban Street Symposium, Seattle, WA, USA, 2007.

[16] F. Garman, O. Luca, and O. Singureanu, "Coping with congestion: shared spaces," Theoretical and Empirical Researches in Urban Management, vol. 7, no. 4, pp. 53-62, 2012.

[17] R. Schönauer, M. Stubenschrott, W. Huang, C. Rudloff, and M. Fellendorf, "Modeling concepts for mixed traffic," Transportation Research Record, no. 2316, pp. 114-121, 2012.

[18] C. Rudloff, R. Schönauer, and M. Fellendorf, "Comparing calibrated shared space simulation model with real-life data," Transportation Research Record, no. 2390, pp. 44-52, 2013.

[19] F. Pascucci, N. C. Rinke, B. Schiermeyer, Friedrich., and V. Berkhahn, "Modeling of shared space with multi-model traffic using a multi-layer social force approach," Transportation Research Procedia, vol. 10, pp. 316-326, 2015.

[20] C. Frosch, Evaluation of Shared Space to Reduce Traffic Congestion: A case study on West Virginia University's Downtown Campus. [M.S. Thesis Civil Engineering], West Virginia University, 2017. 


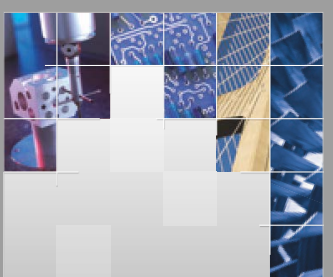

\section{Enfincering}
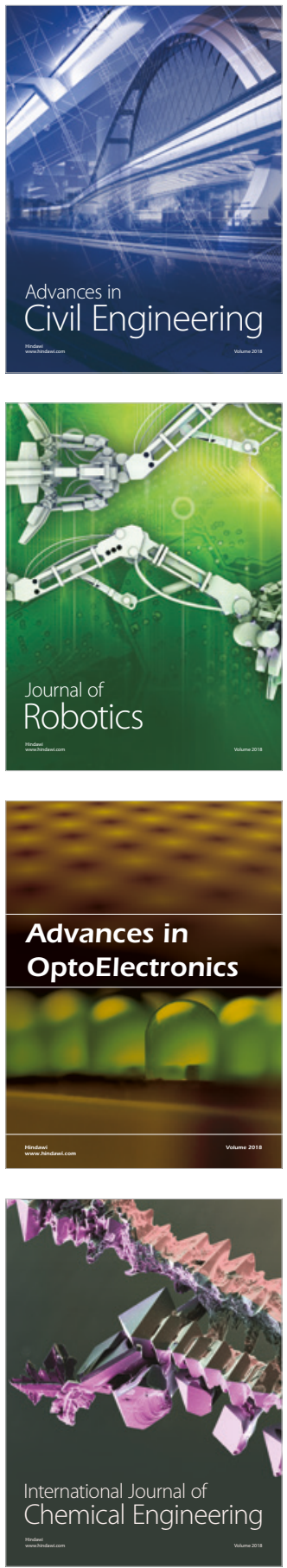

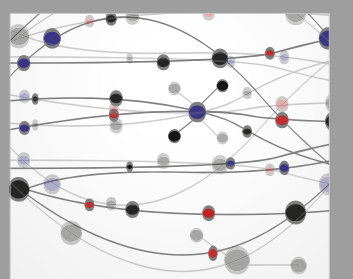

\section{Rotating \\ Machinery}

The Scientific World Journal

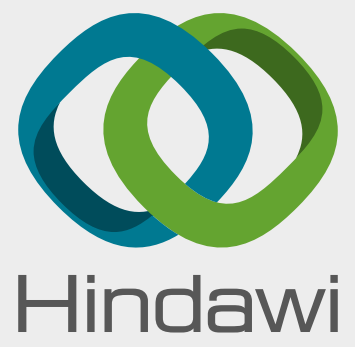

Submit your manuscripts at

www.hindawi.com
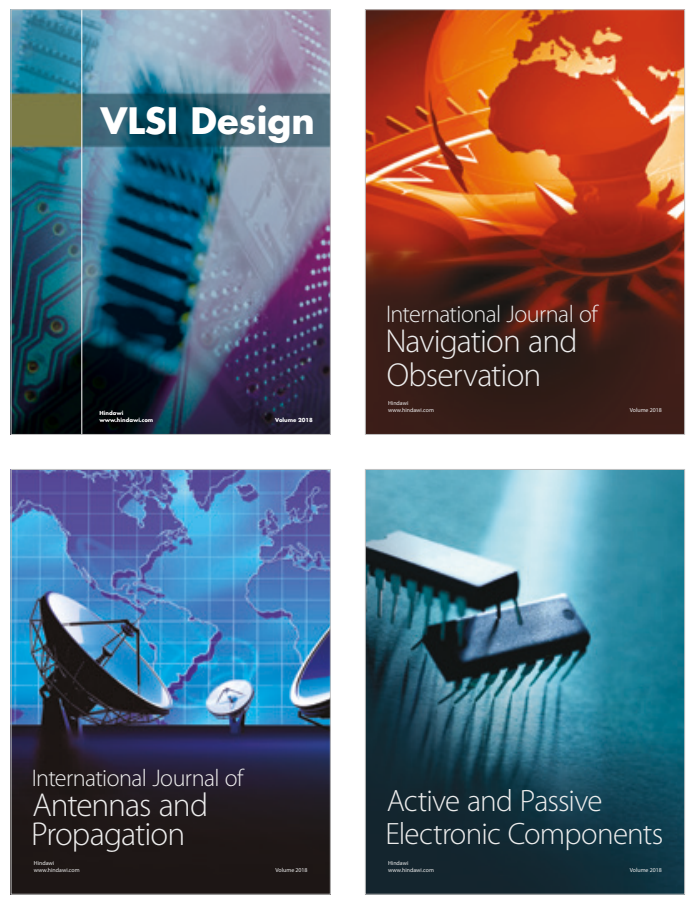
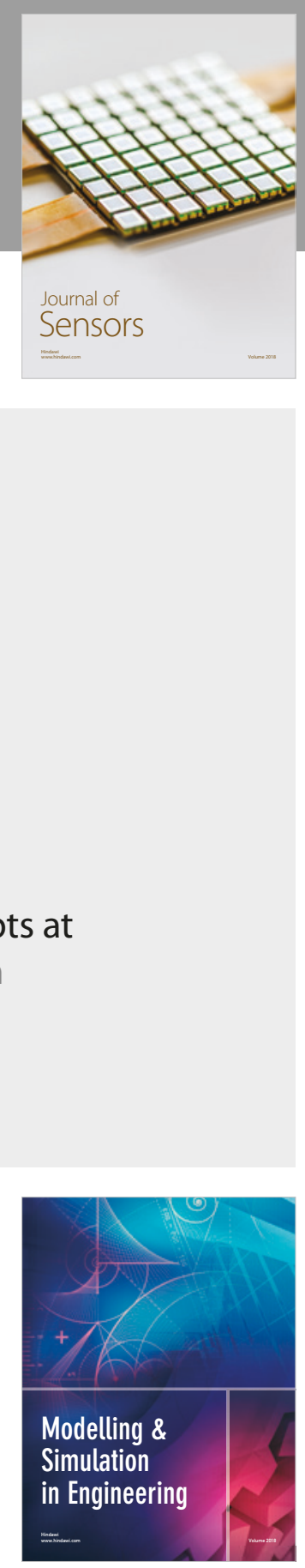

\section{Advances \\ Multimedia}
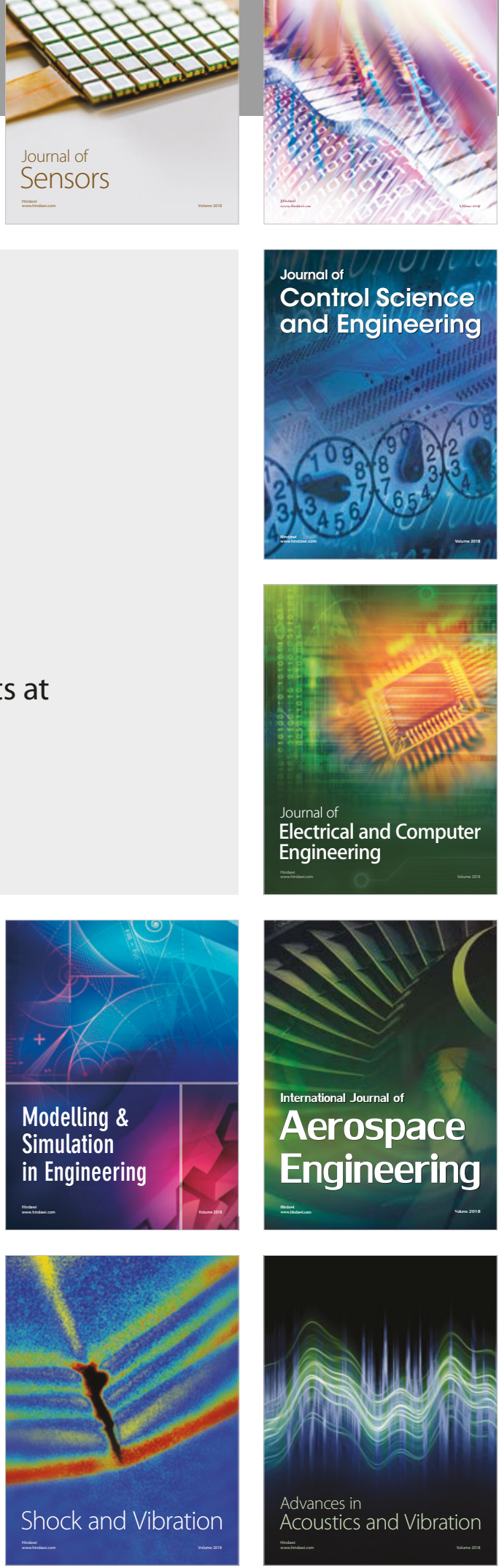